\title{
GPU and CPU Numerical Simulation of Two-Phase Mixtures with Phase Transition
}

\author{
${ }^{1}$ VLADIMIR V. CHUDANOV,${ }^{2}$ ANNA E. AKSENOVA, ${ }^{3}$ ALEXEY A. LEONOV, \\ ${ }^{4}$ ARTEM A. MAKAREVICH \\ 1, 2, 3,4 Nuclear Safety Institute (IBRAE) of Russian Academy of Sciences, 52, Bolshaya Tulskaya street, Moscow, \\ 115191, RUSSIA
}

\begin{abstract}
- the article presents the direct simulation results of two-phase mixtures with phase transition shock tube in dodecane, twophase expansion tube in water and 2D heat and mass transfer in a vertical channel with sodium. For two versions of the code for CPU and GPU calculations possess efficient scaling in the wide range of grid sizes. Scaling for CPU version of the code is close to ideal and does not depend from the grid size and the number of nodes. Efficiency of scaling for GPU version of the code is limited by the size of calculation region per 1 node and could be varied for different models of processor. The value of scaling factor was demonstrated.
\end{abstract}

Keywords - DNS, two-phase, dodecane, sodium, supercomputing.

Received: January 2, 2021. Revised: April 12, 2021. Accepted: April 26, 2021. Published: May 7, 2021.

\section{Introduction}

$N^{i}$ imulation of compressible two-material flows and twophase mixtures concerns with applications in many natural and industrial situations, in astrophysics, physics of explosives, nuclear physics, etc.

In modern conditions, CFD codes are used to simulate such phenomena, and in most cases focused on supercomputer technologies.

Scaling factor is an important characteristic of computing codes, applying parallel approach for numerical calculations. The evaluation of the value of scaling factor for the code, developed for direct numerical simulation of two-phase mixtures with phase transition, is the purpose of this paper. The calculations were performed using the facilities of supercomputers Lomonosov-1 and BlueGene MSU.

Two versions of the code were tested. One is meant for calculations using CPU model of supercomputer, and another one is used for calculations using GPU model of supercomputer. For each code version two groups of numerical tests could be marked. In first group the fixed size of global grid was used, and in second group the size of global grid could be changed.

The value of scaling factor was studied for the two 1D benchmarks: shock tube in dodecane and cavitation in water taking into account phase transition phenomena. Also 2D simulation of the experimental study of heat and mass transfer in a vertical channel with boiling sodium subject to forced lift conditions $[1,2]$ was used for scaling factor evaluation. All runs were succeeding in the scaling close to ideal.

In this paper for the direct simulation of compressible twophase mixture six-equation model [3] was used. Six-equation model allows to circumvent the difficulties arisen from the non-monotonic behavior of the sound speed, which causes inaccuracies in wave transmission across interfaces, from volume fraction variation across acoustic waves, which results in difficulties for the Riemann problem solution, and from the volume fraction positivity problem in the presence of shocks or strong expansion waves. In six-equation model the pressure non-equilibrium approach is developed with following relaxation procedure. It allows constructing non-conservative hyperbolic model with two-energy equations involving relaxation terms. The equations of state and energy conservation on both sides of interfaces are fulfilled.

To account for the heat and mass transfer effects in twophase mixture the temperature and Gibbs free energy relaxation procedures were applied in series as described and proved in [4].

The important critical point concerning the direct numerical simulation of two-phase compressible fluxes is the value of time step forced by the courant limit. The value of the sound speed in liquid phase is usually more, than $10^{3} \mathrm{~m} / \mathrm{s}$, that for typical grids leads to the integration time step less than microsecond. So, the application of parallel technologies for such calculations becomes life-buoy ring. In this paper the value of scaling factor for the code, developed according to parallel algorithm for direct numerical simulation of twophase mixtures with phase transition, is investigated. The calculations were performed using the facilities of supercomputers Lomonosov-1 and BlueGene. Two versions of the code were tested. One is meant for calculations using CPU model of supercomputer, and another one is used for calculations using GPU model of supercomputer. For each code version two groups of numerical tests could be marked. In first group the fixed size of global grid was used, and in second group the size of global grid could be changed. For all runs the scaling close to deal was obtained.

In the first part of the paper the mathematical and numerical model for compressible two-phase mixture simulation are 
described. In the second part the result of simulations for 1D and $2 \mathrm{D}$ numerical tests are presented. The study of scaling factor of the code is reported in the third part of the paper. Some conclusions are summarized in the last fourth part.

\section{Mathematical and numerical models}

To simulate compressible two-phase mixture with nonequilibrium pressures and capillary effects, but without phase transition the following system of equations was accepted [3, $5,6]$ :

$$
\left\{\begin{array}{l}
\frac{\partial \alpha_{g}}{\partial t}+\vec{u} \nabla \alpha_{g}=\mu\left(p_{g}-p_{l}\right) \\
\frac{\partial \rho}{\partial t}+\operatorname{div}(\rho \vec{u})=0 \\
\frac{\partial\left(\rho Y_{l}\right)}{\partial t}+\operatorname{div}\left(\rho Y_{l} \vec{u}\right)=0 \\
\frac{\partial \vec{w}}{\partial t}+\operatorname{div}(\vec{w} \vec{u})=0 \\
\frac{\partial(\rho \vec{u})}{\partial t}+\operatorname{div}(\rho \vec{u} \otimes \vec{u}-T)=0, \\
T=-\left(p+\lambda \frac{m-1}{m}|\vec{w}|^{m}\right) I+\lambda|\vec{w}|^{m} \times\left(I-\frac{\vec{w}}{|\vec{w}|} \otimes \frac{\vec{w}}{|\vec{w}|}\right) \\
\frac{\partial\left(\alpha_{g} \rho_{g} e_{g}\right)}{\partial t}+\operatorname{div}\left(\vec{u} \alpha_{g} \rho_{g} e_{g}\right)+\alpha_{g} p_{g} \operatorname{div} \vec{u}=-P_{l} \mu\left(p_{g}-p_{l}\right) \\
\frac{\partial\left(\alpha_{l} \rho_{l} e_{l}\right)}{\partial t}+\operatorname{div}\left(\vec{u} \alpha_{l} \rho_{l} e_{l}\right)+\alpha_{l} p_{l} \operatorname{div} \vec{u}=P_{l} \mu\left(p_{g}-p_{l}\right)
\end{array}\right.
$$

The following notations in equation system (1) were used:

$\vec{u}$-velocity,

$\alpha$-volume fraction,

$p$ - pressure,

$\rho$ - density,

$e$ - specific energy,

indexes $g, l$ denote thermodynamic variables of gas, liquid phase, respectively;

$\rho=\alpha_{g} \rho_{g}+\alpha_{l} \rho_{l}$

$Y_{l}=\frac{\alpha_{l} \rho_{l}}{\rho}$;

$w=\nabla Y_{l}$

$\mu$ - pressure relaxation coefficient;

$\lambda$ - capillarity parameter;

$m$ - interface sharpness parameter ( $m=1$ for sharp interfce);

$P_{I}=\frac{z_{l} p_{g}+Z_{g} p_{l}}{z_{l}+z_{g}}-$ interface pressure,

$Z_{g}=\rho_{g} c_{g}, Z_{l}=\rho_{l} c_{l}$.

To close system (1) stiffened gas equation of state (SGEOS) is used. An essential issue is that the various SG-EOS parameters are linked to each other to fulfill some constraints to recover the phase diagram [7]. This makes such a choice of EOS suitable for phase transitions.

To account for phase transition the equilibrium evaporation model is used. In this model the phase heat exchange and phase transition processes lead two-phase mixture in the state with equal values of temperatures and Gibbs free energies.
This allows defining the values of thermodynamic parameters after phase exchanges using the procedures of instantaneous relaxation for temperatures and for Gibbs free energies.

At first the procedure of temperatures relaxation, retaining the pressures equality, is applied. And then the procedure of free Gibbs energies relaxation, retaining the pressures and temperatures equality, is used. Each relaxation procedure involves the iterative algorithm [4].

The equilibrium evaporation model is realized including specific source terms in the system (1):

$$
\left\{\begin{array}{l}
\frac{\partial \alpha_{g}}{\partial t}=\frac{Q}{k}+\frac{\dot{m}}{\rho_{I}} \\
\frac{\partial\left(\alpha_{g} \rho_{g}\right)}{\partial t}=\dot{m} \\
\frac{\partial\left(\alpha_{l} \rho_{l}\right)}{\partial t}=-\dot{m} \\
\frac{\partial w_{x}}{\partial t}=0 \\
\frac{\partial w_{y}}{\partial t}=0 \\
\frac{\partial w_{z}}{\partial t}=0 \\
\frac{\partial\left(\rho u_{x}\right)}{\partial t}=0 \\
\frac{\partial\left(\rho u_{y}\right)}{\partial t}=0 \\
\frac{\partial\left(\rho u_{z}\right)}{\partial t}=0 \\
\frac{\partial\left(\alpha_{g} \rho_{g} e_{g}\right)}{\partial t}=Q+e_{I} \times \dot{m} \\
\frac{\partial\left(\alpha_{l} \rho_{l} e_{l}\right)}{\partial t}=-Q-e_{I} \times \dot{m}
\end{array}\right.
$$

The following notations in equation system (2) were used: $Q=\theta \times\left(T_{g}-T_{l}\right)$ is heat transfer term, $\theta \rightarrow \infty$;

$k$ is defined using the condition $\frac{\partial p_{g}}{\partial t}=\frac{\partial p_{l}}{\partial t}$ in temperatures relaxation procedure;

$\rho_{I}, e_{I}$ are defined using the conditions $\frac{\partial p_{g}}{\partial t}=\frac{\partial p_{l}}{\partial t}$ and $\frac{\partial T_{g}}{\partial t}=\frac{\partial T_{l}}{\partial t}$ in Gibbs free energies relaxation procedure;

$\dot{m}$ is mass rate coefficient, which is calculated from the requirement, that the difference of Gibbs free energies at the end of numerical time step is vanishing [8].

The explicit numerical algorithm to simulate two-phase mixture with non-equilibrium pressures and capillary effects and with phase transition consists from two steps according splitting approach.

At first step system (1) is resolved using HLLC Riemann solver [9] with subsequent pressure relaxation procedure [3]. Then new values of thermodynamic variables are used as input parameters for solving system (2). 


\section{Numerical Predictions}

\subsection{Test-1: Two-phase shock tube in dodecane}

The tube of $1 \mathrm{~m}$ length is filled with liquid dodecane under high pressure at the left, and with the vapor dodecane at atmospheric pressure at the right. The initial discontinuity is set at $0.75 \mathrm{~m}$, and the initial data are:

- At left $p_{\text {left }}=10^{8} \mathrm{~Pa} ; \rho_{\text {left }}=500 \mathrm{~kg} / \mathrm{m}^{3} ; u_{\text {left }}=0 \mathrm{~m} / \mathrm{s}$.

- At right $p_{\text {right }}=10^{5} \mathrm{~Pa} ; \rho_{\text {right }}=2 \mathrm{~kg} / \mathrm{m}^{3} ; u_{\text {right }}=0$ $\mathrm{m} / \mathrm{s}$

In each side of the shock tube the presence of a small volume fraction of the other fluid, typically $10^{-6}$, is allowed for numerical reasons. The results of calculations with and without phase transition for gas stiffened EOS are presented in Fig. 1 at time $t=473 \mu$ s. The results were obtained for grid size 1024 .
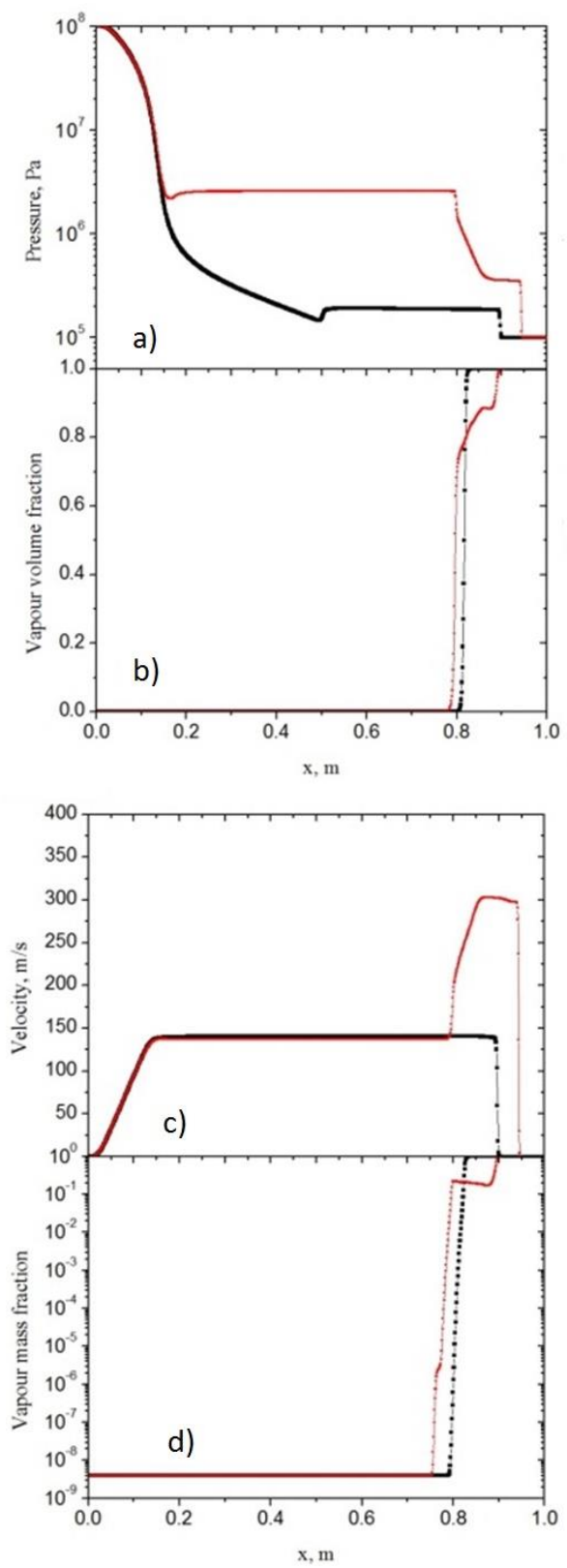

Fig. 1 two-phase shock tube in dodecane
At figure 1 dodecane liquid-vapor shock tube without phase transition is shown by black color and one with phase transition is shown by red color.

In test with phase transition extra wave appears between the rarefaction wave and the contact discontinuity which corresponds to the evaporation front. Indeed, rarefaction waves propagate through the liquid producing a superheated liquid and evaporation has occurred. An extra wave representing the evaporation front propagates through the superheated liquid and produces a liquid vapor mixture at thermodynamic equilibrium with a high velocity [8].

\subsection{Test-2: Two-phase expansion tube in water}

Consider a $1 \mathrm{~m}$ tube filled with liquid water at atmospheric pressure and with density $\rho=1150 \mathrm{~kg} / \mathrm{m}^{3}$. A weak volume fraction of vapor $(\alpha=0.01)$ is initially added to the liquid. The initial discontinuity is set at $0.5 \mathrm{~m}$. The left velocity is $-2 \mathrm{~m} / \mathrm{s}$ and the right velocity is $2 \mathrm{~m} / \mathrm{s}$.

In this test a small time step is required to obtain a stable solution $(\mathrm{CFL} \approx 0.03)$. The small time here indicates that there is a stiffness coming from the relaxation procedures [4].

Fig. 2 presents the solution when the phase transition is involved and is compared with the solution without phase transition at $t=3.2 \mathrm{~ms}$.

The two-phase expansion tube involves two expansion waves. The vapor volume fraction increases at the center of the domain due to the gas mechanical expansion present in small proportions. The rarefaction waves make the liquid metastable and phase transition has to be added. Liquid water is expanded until the saturation pressure is reached then evaporation appears and quite small of vapor is created.

At figure 2 water liquid-vapor expansion tube with phase transition at $\mathrm{t}=3.2 \mathrm{~ms}$ is shown.

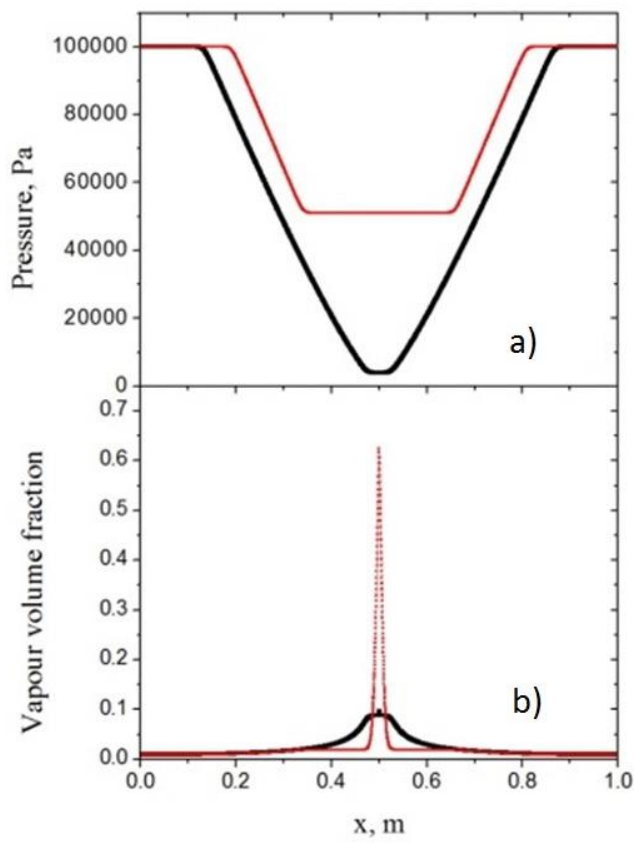

Fig. 2 test two-phase expansion tube in water 


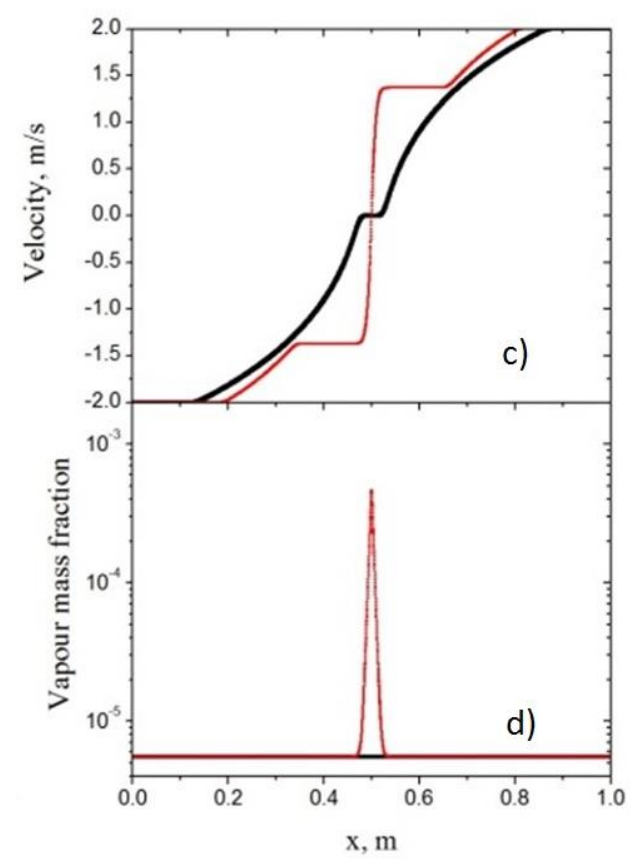

Fig. 2 test two-phase expansion tube in water (continue)

The computed results with phase transition are shown by red color. They are compared with the results without phase transition, which are shown by black color. The results were obtained for grid size 4096 .

In both cases, Test- 1 and Test- 2 were obtained a good qualitative coincidence with the data [8] and [4]. No quantitative results were presented in these papers, so no comparison was made. To demonstrate the possibilities of qualitative prediction of the results with the help of the developed codes, modeling of two-dimensional heat and mass transfer in a vertical channel with sodium was performed.

\subsection{D heat and mass transfer in a vertical channel with sodium}

The vertical dimension of the working region of a flat channel with transverse size of $5 \mathrm{~mm}$ is $70 \mathrm{~cm}$. The heat flux through the side walls was assumed to be $\sim 0.57 \mathrm{MW} / \mathrm{m}^{2}$. The initial volume fraction of sodium vapor in the whole pipe is $10^{-5}$, and the initial temperature of two-phase sodium mixture was assumed equal to $1153 \mathrm{~K}$ everywhere. This value of temperature is $3 \mathrm{~K}$ less than the boiling point at atmospheric pressure. The distribution of the initial vertical velocity corresponds to a mass flow of $166 \mathrm{~kg} / \mathrm{m}^{2} / \mathrm{s}$. At the lower boundary, constant values of temperature and volume fraction are maintained, equal to the initial values. A constant mass flow is also maintained at the lower boundary. At the upper boundary, atmospheric pressure is fixed.

A vertical profile of the pressure averaged over the cross section at time instant $0.22 \mathrm{~s}$ is shown in Fig. 3 .

The points correspond to the experimental values of the pressure for two different values of the heat fluxes [1]. The dashed curve corresponds to the interpolation of the experimental values for the heat flux of $0.57 \mathrm{MW} / \mathrm{m}^{2}$ assuming a linear dependence of the pressure values on the heat flux.

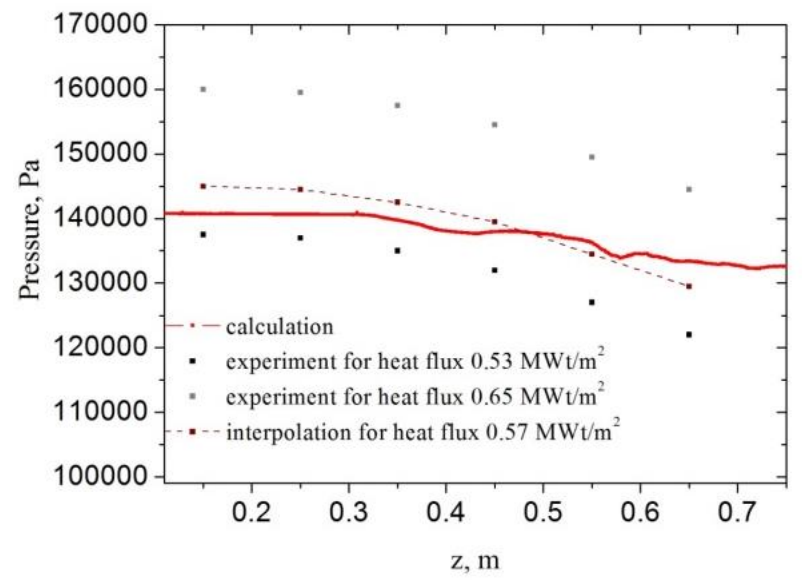

Fig. 3 pressure distribution along the channel

A good qualitative agreement of the calculated and experimental values is obtained. The difference in the magnitude of the pressure difference over the working region of the pipe can be related to the "flat" approximation of the model calculation, which leads to different values of the ratio of the transverse length of the heating section to the enclosed area.

2D profiles of vapour volume fractions for different time instants are shown in fig.4. The process of vapour film generation is observing.
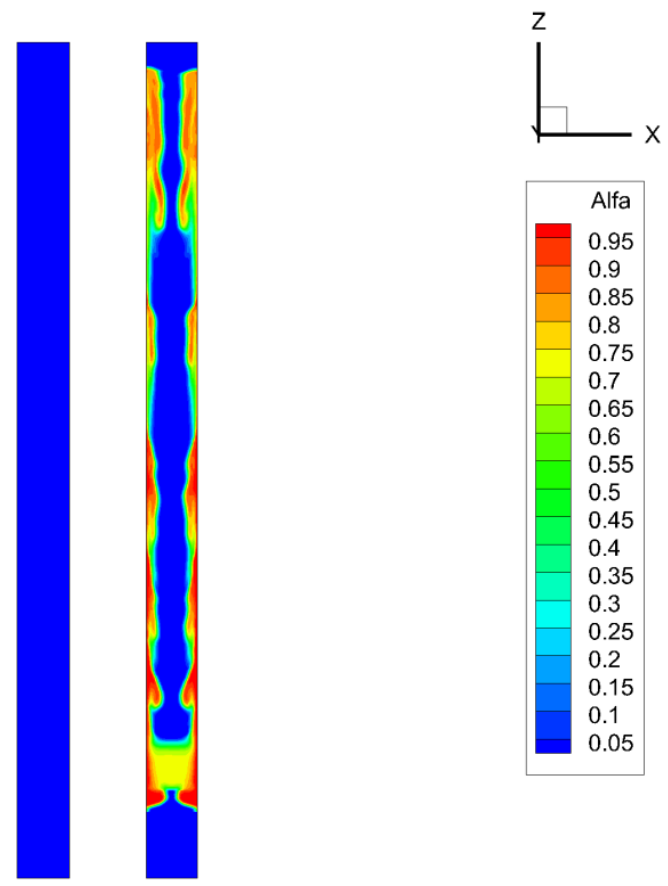

Fig. 4 profile of vapour volume fraction for time instants 0 and $0.21 \mathrm{~s}$ 


\section{Investigation of Scaling Factor}

As it had been noticed above the application of parallel technologies for direct numerical calculations of two-phase mixtures is highly motivated. From this point two parallel FORTRAN versions of the code concerning direct numerical simulation of two-phase mixtures with phase transition were processed for CPU and GPU facilities of supercomputers.

In GPU version of the code OpenAcc programming technology for PGI Fortran compiler was used. Scaling factor of the code had investigated using supercomputers Lomonosov-1 and BlueGene MSU. The characteristics of these supercomputers are described in table 1 . CPU version of the code was tested on both supercomputers, and GPU version had been run on Lomonosov-1 supercomputer only.

Tab. 1 the characteristics of Lomonosov-1 and BlueGene MSU supercomputers

\begin{tabular}{|c|c|c|}
\hline \multicolumn{3}{|c|}{ Lomonosov-1 } \\
\hline \multicolumn{3}{|c|}{ web resource http://users.parallel.ru/wiki/pages/22-config } \\
\hline \multirow[t]{3}{*}{ CPU } & model & $\begin{array}{l}\text { Intel Xeon X5570 } \\
2 \text { processors from } 4 \text { cores, } \\
8 \text { CPU cores per node }\end{array}$ \\
\hline & memory & $12 \mathrm{~GB}$ \\
\hline & nodes & 4096 \\
\hline \multirow[t]{3}{*}{ GPU } & model & $\begin{array}{l}\text { Intel Xeon E5630 } \\
\text { graphic accelerators per node, } \\
8 \text { CPU cores per node }\end{array}$ \\
\hline & memory & $24 \mathrm{~GB}$ \\
\hline & nodes & 830 \\
\hline \multicolumn{3}{|c|}{ BlueGene MSU } \\
\hline \multicolumn{3}{|c|}{ web resource http://hpc.cmc.msu.ru/bgp } \\
\hline \multirow[t]{3}{*}{ CPU } & model & $\begin{array}{c}\text { PowerPC } 450 \\
\text { processor from } 4 \text { cores, } \\
4 \text { CPU cores per node }\end{array}$ \\
\hline & memory & $2 \mathrm{~GB}$ \\
\hline & nodes & 2048 \\
\hline
\end{tabular}

At first the range of grid sizes for effective scaling was investigated. Here the acceleration coefficient for 1 MPI process is defined as ratio of run time of 1 iteration on maximum available grid to the run time of 1 iteration on fixed grid. The ideal dependence, defined as equal proportionality between run time and grid size, is also shown as stroked line. For CPU version of the code grid size does not influence significantly on the code efficiency.

For each diminishing of grid size the run time decreases in the same proportion. For GPU version the grid size effects on the code efficiency heavily. For effective calculations using GPU facility of supercomputer Lomonosov-1 it is necessary to employ the grid sizes not less than $20 \%$ from maximum available grid size. For smaller grid sizes the efficiency of the
GPU version of the code is decreased. This conclusion could be changed if using other modern versions of GPU processors.

Then the value of scaling factor was estimated in strong and weak senses. In Fig.5 the dependences of acceleration coefficient from the number of used nodes on fixed grid are shown for CPU and GPU versions.

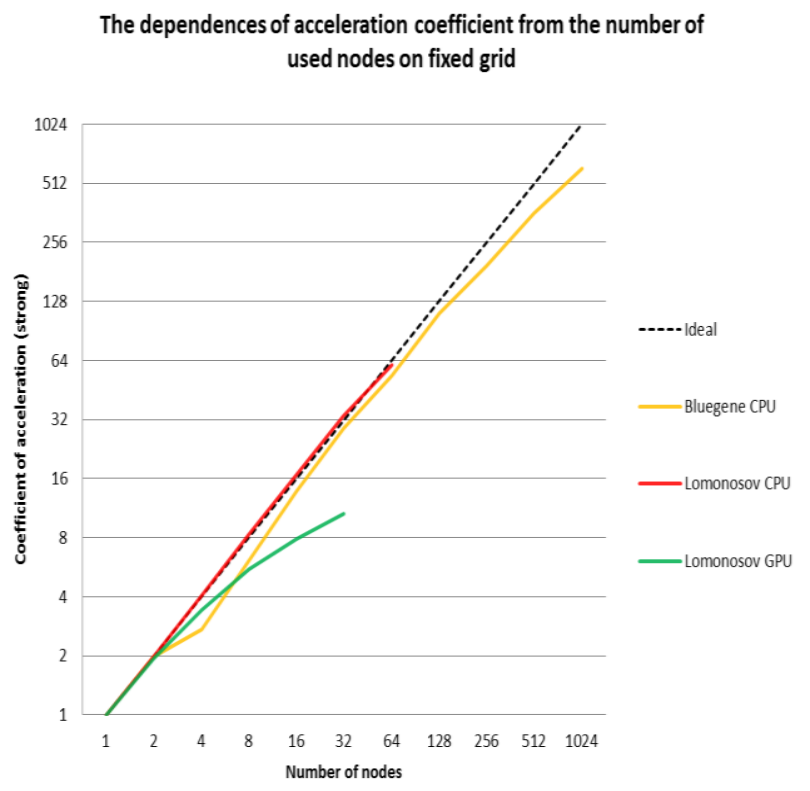

Fig. 5 the dependences of acceleration coefficient from the number of used nodes on fixed grid

In this strong definition for fixed grid the acceleration coefficient is defined as ratio of run time of 1 iteration on 1 node to the run time of 1 iteration on $\mathrm{N}$ nodes. The ideal dependence, where coefficient of acceleration equals number of nodes, is also shown as stroked line.

For CPU version of the code the amount of data transmitted between the nodes does not affect the run time if the number of nodes does not reach saturation state.

Efficiency of GPU version of the code depends from the number of nodes in the same manner as from the grid size on 1 MPI process. While grid size on 1 MPI process is more, than $20 \%$ from maximum available grid size, the coefficient of acceleration is close to ideal. If the number of nodes is farther increasing then the efficiency of GPU version could be decreased.

In Fig. 6 the dependences of acceleration coefficient from the number of used nodes on fixed grid for 1 node are shown for CPU and GPU versions.

In this weak definition with fixed grid for 1 node the acceleration coefficient is defined as ratio of run time for 1 iteration on one node multiplying number of nodes to the run time of 1 iteration on $\mathrm{N}$ nodes. For GPU version two variants of global grid size increasing were applied.

In first variant only one space direction to increase the calculation region was used. In this variant the amount of data exchanges among the neighboring nodes is constant. 
WSEAS TRANSACTIONS On HEAT and MASS TRANSFER
Vladimir V. Chudanov, Anna E. Aksenova, Alexey A. Leonov, Artem A. Makarevich

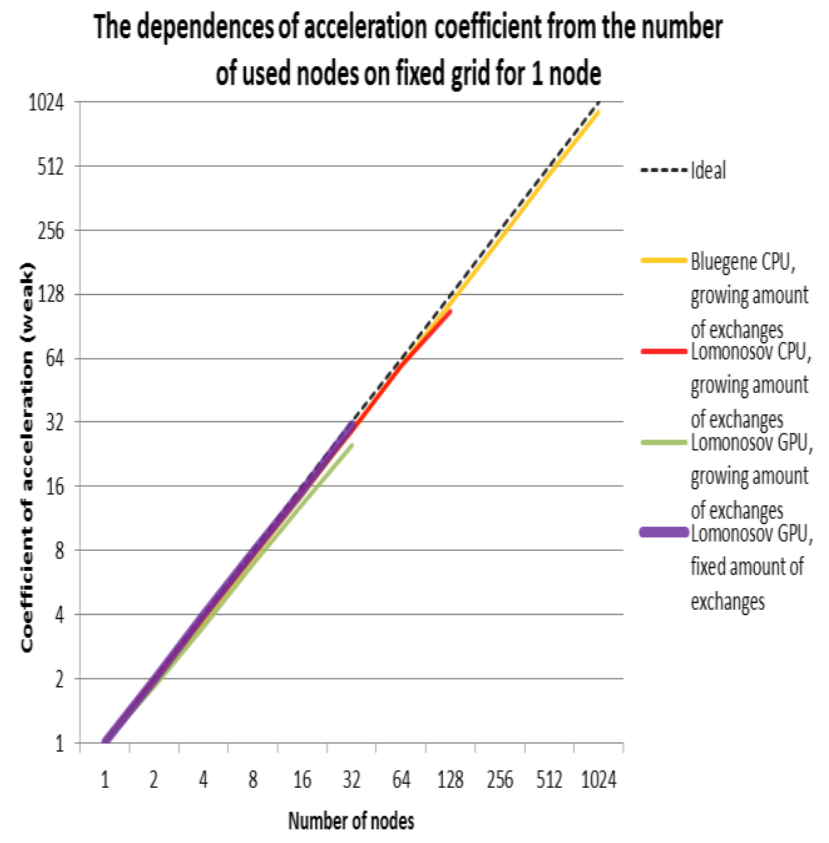

Fig. 6 the dependences of acceleration coefficient from the number of used nodes on fixed grid for 1 node

In second variant the increasing of global grid size was realized for all space directions. In last variant the amount of data exchanges among the neighboring nodes is increased with the size of global grid. The ideal dependence, where coefficient of acceleration equals number of nodes, is also shown as stroked line. CPU version of the code possesses strong and weak scaling. The scaling of GPU version of the code is limited by amount of data transmitted between nodes.

\section{Conclusion}

The value of scaling factor for the code, processed for direct numerical simulation of two-phase mixtures with phase transition, was studied for the two 1D benchmarks: shock tube in dodecane and cavitation in water taking into account phase transition phenomena.

Also 2D simulation of the experimental study of heat and mass transfer in a vertical channel with boiling sodium subject to forced lift conditions was used for scaling factor evaluation.

As a whole, two versions of the code for CPU and GPU calculations possess efficient scaling in the wide range of grid sizes. Scaling for CPU version of the code is close to ideal and does not depend from the grid size and the number of nodes. Efficiency of scaling for GPU version of the code is limited by the size of calculation region per 1 node and could be varied for different models of processor.

\section{Acknowledgment}

The research is carried out using the equipment of the shared research facilities of HPC computing resources at Lomonosov Moscow State University.

\section{References}

[1.] Yu. Zeigarnik, V. Litvinov, "Investigation of pressure loss of sodium boiling in a tube", High Temp., 5, 1977, p.1116.

[2.] A. Aksenova, V. Chudanov, A. Leonov, A. Makarevich, "Direct numerical simulations of two-phase gas dynamic flows with phase transitions for water and for liquid sodium", MATEC Web of Conferences, 115, №05009, 2017, p.1.

[3.] R. Saurel, F. Petitpas, Ray A. Berry, "Simple and efficient relaxation methods for interfaces separating compressible fluids, cavitating flows and shocks in multiphase mixtures", Journal of Computational Physics, 228, 2009, p.1678.

[4.] A. Zein, M. Hantke, G. Warnecke, "Modeling phase transition for compressible two-phase flows applied to metastable liquids", Journal of Computational Physics, 229, №8, 2010, p.2964.

[5.] Ray A. Berry, R. Saurel, F. Petitpas, E. Daniel, O. Le Metayer, S. Gavrilyuk, N. Dovetta, R.C. Martineau, "Progress in the Development of Compressible, Multiphase Flow Modeling Capability for Nuclear Reactor Flow Applications", Report Idaho National Laboratory, INL/EXT-08-15002, 2008.

[6.] V. Chudanov, A. Aksenova, A. Leonov, "Unified CFD approach to simulate two-phase flows in presence of surface forces", International Journal of Mathematical Models and Methods in Applied Sciences, 8, 2014, p.248.

[7.] O. Le Metayer, J. Massoni, R. Saurel, Elaborating equations of state of a liquid and its vapor for two-phase flow models, Int. J. Therm. Sci., 43, №3, 2004, p.265.

[8.] R. Saurel, F. Petitpas, R. Abgrall, "Modelling phase transition in metastable liquids: application to cavitating and flashing flows", Fluid Mech., 607, 2008, p.313.

[9.] E. Toro, M. Source, W. Spears, "Restoration of the contact surface in the HLL-Riemann solver", Shock Waves, 4, №1, 1994, p.25.

\section{Creative Commons Attribution License 4.0 (Attribution 4.0 International, CC BY 4.0)}

This article is published under the terms of the Creative Commons Attribution License 4.0 https://creativecommons.org/licenses/by/4.0/deed.en_US 